\title{
Variability in Systemic Treatment Efficacy for Onychomycosis: Information That Clinical Studies Do Not Impart to the Office Dermatologist
}

\author{
Avner Shemer $^{\mathrm{a}, \mathrm{b}}$ Ralph Daniel ${ }^{\mathrm{c}}$ Dimitris Rigopoulos ${ }^{\mathrm{d}}$ Renata Farhi $^{\mathrm{e}}$ \\ Meir Babaev ${ }^{f}$

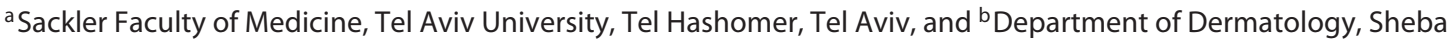 \\ Medical Center, Tel Hashomer, Ramat Gan, Israel; ' University of Mississippi Medical Center and University of \\ Alabama, Birmingham, AL, USA; ${ }^{d}$ 2nd Department of Dermatology and Venereology, National and Kapodistrian

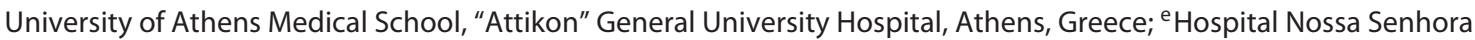 \\ da Saúde, University Fundação Tecnico Educacional Souza Marques, Rio de Janeiro, Brazil; fDivision of Medicine, \\ Faculty of Health Sciences, Ben-Gurion University of the Negev, Beer Sheva, Israel
}

\section{Keywords}

Nail disease $\cdot$ Onychomycosis · Treatment efficacy $\cdot$ Clinical studies

\begin{abstract}
Wide differences in the cure rates of onychomycosis in various clinical studies can be avoided if we bear in mind some insights that will help us make study results more consistent. We suggest less rigid criteria in defining the cure rate, the inclusion of all toenails in studies, and the exclusion of nails with a history of trauma. Rather than address patients as a homogenous group, regardless of age, we suggest dividing them into age groups. Researchers should not forget inflammatory diseases that can imply changes on the nails and should take into account the duration of onychomycosis, nail changes caused by asymmetry, and the possibility of dermatophytoma.
\end{abstract}

(c) 2017 S. Karger AG, Basel

In searching the literature regarding onychomycosis, clinical studies have shown a significant difference in clinical and mycological results. Although one does not expect all studies to be the same, variations in study design might also cause some disparities in the cure rate. While variations in results are plausible, a considerable range - between 14 and 90\% - is unacceptable [1-3].

When we design a clinical study or review the methods of a clinical study, many insights should be kept in mind. Those insights will help researchers to avoid gaps that can be noticed throughout clinical studies. A review of multiple clinical studies reveals several variations in the definition of clinical cure, and clinical cure rates vary between 80 and $100 \%$ in different onychomycosis studies.

In the literature, both clinical and mycological cure definitions have strict and less rigid criteria. Clinical cure is defined as $100 \%$ improvement in strict criteria and as $80 \%$ improvement in less rigid criteria. Mycological cure is defined according to strict criteria as negative culture and negative direct microscopy. Less rigid criteria for mycological cure include negative culture and do not take into consideration direct microscopy. The application of strict and less rigid criteria makes a significant difference in study outcomes [4]. The logical approach is that of the less rigid criteria, where the culture must be negative and direct microscopy may be either negative or positive. The expla-

\section{KARGER}

(c) 2017 S. Karger AG, Basel

E-Mail karger@karger.com

www.karger.com/sad
Avner Shemer, MD

Lev Yasmin Medical Center

18 Pinhas Lavon St.

Netanya 42701 (Israel)

E-Mail ashemer1@gmail.com 
nation of less rigid criteria, where positive direct microscopy might be allowed, is that dead and live hyphae cannot be distinguished under the standard light microscope used in typical mycology laboratories. A systemic antifungal medication may inhibit or kill the fungus, but it will not eliminate the hyphae. Dead hyphae in direct microscopy cannot be interpreted as an active fungal infection.

To date, the great toe nail - the hallux - serves as the target nail, and this target nail plays a critical role with respect to the extent of the complete cure rate of tested systemic antifungal drugs. When patients ask about the odds of a cure, the physician will refer to figures based on data related to the great toe nail alone. Usually, the other involved toenails are not considered.

The great toe nail is usually chosen for an evaluation of the efficacy of antifungal agents because it is more suitable for measurements of the involved versus healthy nail areas. As the tested area is larger, the calculation is easier and more precise.

Why might the inclusion of the first toenail without the other toenails lead to erroneous conclusions? Usually, the great toe nail is prone to different types of trauma, especially repeated minor trauma, of which even the patient may not be aware. Major trauma, as well as repeated minor trauma, may cause irreversible nail deformity Successful systemic antifungal treatment may eliminate the fungal component and its reversible contributions to nail plate deformation, but the irreversible mechanical matrix damage cannot be eliminated, and the nail may remain deformed.

Study results show that the potential of the clinical complete cure rate of the great toe nail is lower compared to the second, third, and fourth toenails. The second toenail achieved the best clinical results [5].

\section{Wide Age Variability}

To date, the inclusion criteria regarding age are "above 18 years up to 70 years old" [6-9]. This means 18 -year-old and 70-year-old onychomycosis patients will be evaluated in the same way and assigned to the same study group. Clinical and mycological cure rates are lower in older age groups due to slower nail growth rate $[10,11]$. Moreover, there is a statistical correlation between age and severity of the onychomycosis [12]. Children and young adults usually have mild to moderate onychomycosis, while adults and the elderly have moderate to severe onychomycosis [7, 13]. Nevertheless, untreated children and young adults will proceed over time to develop moderate and severe forms of onychomycosis. This gradual wors- ening occurs due to the further spread of a fungal infection, as well as nail matrix damage from retrograde mechanical pressure applied by a thickened nail and ill-fitting shoes. To generate more precise efficacy data that reflect everyday dermatology practice, it is advisable to divide the participants into narrower age groups.

\section{Exclusion of Traumatic Nails}

Nails that were deformed by mechanical injury are not usually excluded from onychomycosis clinical studies [7, 14]. A damaged matrix will always create a deformed nail plate, and we have practically two components that contribute to a damaged nail plate: onychomycosis and mechanical matrix damage. The patients usually can remember major traumatic events, but may be unaware of minor repetitive trauma or old major trauma. To prevent speculations about the origin of nail deformation, we must avoid including nails that underwent mechanical trauma. In everyday practice, there is no tool available to measure what percentage of the deformation was caused by mechanical damage and what percentage was caused by fungal infection. The fifth toenail, especially in women who wear tight shoes, is often deformed. Hence, this nail should also be excluded from studies [14].

\section{Bearing in Mind Inflammatory Skin Diseases}

Some patients have a combination of onychomycosis and an inflammatory skin disease, such as psoriasis, lichen planus, etc. Nail changes due to inflammatory skin diseases are theoretically listed in exclusion criteria; however, in the real-life practice, it is difficult to distinguish between such changes and pure onychomycosis. Successful treatment may sometimes eliminate the fungal elements and achieve a mycological cure, but not a clinical cure [4]. In psoriatic patients, several nails could be involved or one nail might have psoriasis alone or psoriasis combined with onychomycosis, while the adjacent nail might be healthy but infected with fungus [15].

\section{Duration of Onychomycosis}

In the majority of clinical studies, there is no time limitation of onychomycosis duration, which is an important variable. Retrograde continuous pressure from the inner part of the shoe is greater in onychomycosis, and pro- 
longed pressure contributes to the mechanical component of nail deformation $[7,14]$. The addition of longstanding onychomycosis to the exclusion criteria in clinical studies may contribute to results that are more realistic. We recommend excluding patients with disease duration longer than 30 years.

\section{Using a Precise Scoring System}

In the past, a simplified classification system was used for onychomycosis severity scoring determined by a percentage of involvement. Less than 50\% involvement was defined as mild, $51-75 \%$ involvement as moderate, and above $75 \%$ as severe [16-18]. According to this classification, a mild $10 \%$ involvement and $50 \%$ involvement are in the same category, despite the fact that $10 \%$ involvement is much easier to treat and better results are achieved. Some confusion can arise in severe groups, as well; for example, 75\% involvement has a much higher chance of achieving a complete cure compared to $100 \%$ involvement, which is total dystrophic onychomycosis with a slim chance of a complete cure. In 2011, an Onychomycosis Severity Index (OSI) was suggested by Carney and colleagues [19]. The new classification is much more precise and easier for calculations. The OSI scoring system includes dividing the nail plate into five parallel horizontal lines and by adding dermatophytoma or a thick nail plate. The inclusion of both mild and severe cases in the same study may lead to a significant bias. It is well known that the more severe the onychomycosis, the less complete the cure that can be achieved [20]. Clear inclusion and exclusion criteria should be applied to separate mild and severe cases into distinct study groups.

\section{Dermatophytoma and Thick Nails}

Dermatophytoma is an abundance of fungal filaments compacted and forming a fungal ball of biofilm [21]. The diagnosis of dermatophytoma can be difficult to conclude for dermatologists without enough experience or for those who have no experience with onychomycosis. Dermatophytoma is characterized clinically by single or multiple white or yellow bands on the nail plate (Fig. 1). It is resistant to systemic antifungal treatment and requires chemical or surgical avulsion [22]. When nails with dermatophytoma are included in studies by mistake, there may be a cure for all onychomycotic nails except the spe-

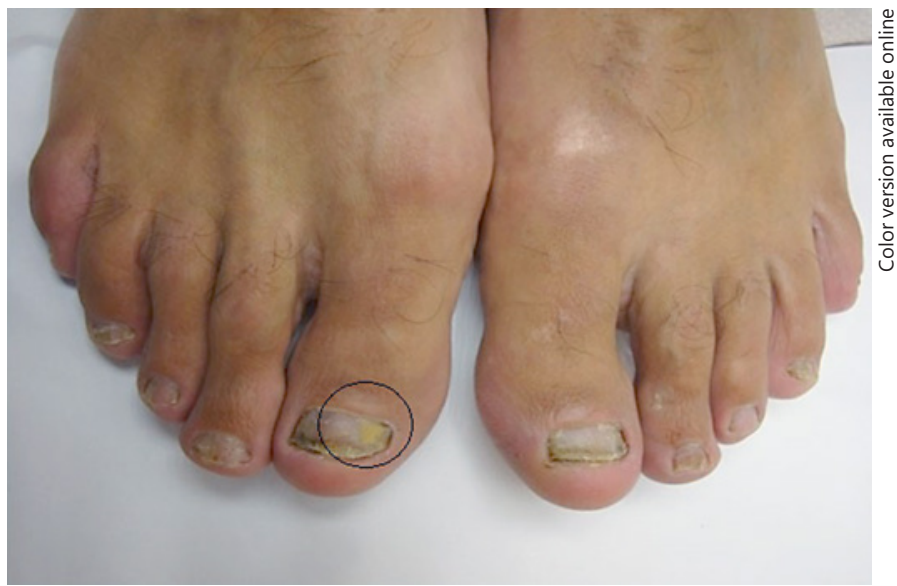

Fig. 1. Dermatophytoma. Note the distinct yellow band on the right toenail.

cific area of the nail with dermatophytoma. In this case, the clinical cure of the nail with dermatophytoma will be interpreted as a failure.

A thick nail is a genetic tendency to create thick and somehow deformed nails. In patients with thick nails combined with onychomycosis, systemic antifungal treatment may eliminate the fungus, but not the thickness, and this may be interpreted as a failure, even though a mycological cure was achieved. Antifungal medication will not cure the thick nail. Moreover, many times, young dermatologists who are usually responsible for conducting studies mistakenly interpret the thick nail as onychomycosis.

\section{Coexistence of Pathogens}

Rarely, two different pathogens may present simultaneously in the same nail. For a diagnosis of nondermatophyte molds (NDM) and yeasts, it is recommended to use Sabouraud's dextrose agar with cycloheximide. Some yeasts and NDM may be resistant to terbinafine or itraconazole. Terbinafine can achieve a mycological cure in a Trichophyton infection, but NDM and Candida spp. can be resistant to terbinafine.

\section{AGNUS Phenomenon}

There are skeletal asymmetries, for example, differences in feet length. Such asymmetries change foot biodynamics and lead to onycholysis, nail plate changes, and 
nail bed keratosis resembling onychomycosis. Asymmetric gait nail unit syndrome (AGNUS) is the result of asymmetric mechanical pressure that causes dermatophyte-free asymmetric nail deformations [23, 24]. Asymmetric nail damage that does not respond to antimycotic treatment leads to different cure rates in every toenail.

In conclusion, if we achieved a partial cure of some nails after systemic antifungal therapy and we ask ourselves whether that is a cure or a failure, the answer is, "It depends on which nail we are discussing." The correct answer to our question should be, "If one or more nails achieved a complete clinical and mycological cure, this is proof of therapy effectiveness." Regarding the nails that did not respond, we could assume there is another problem that made curing impossible. Hence, we can specify the cure rate of a therapy even if we have cured only a single nail. In the rest of nails that were not cured, we assume the existence of an issue unrelated to the fungal in- fection or a combination of disorders that was not to be solved by antimycotic therapy from the beginning.

It is possible that nails that did not achieve a complete cure had a deformation unrelated to the fungal infection. Another possibility is a combination of the fungal infection and another deformation. A positive mycology from such a nail may mislead us to think we have purely onychomycosis. Successful antifungal therapy can eliminate the fungal component, as well as the reversible deformation damage that was caused by the fungal infection. However, antifungal therapy cannot influence a deformation that was caused by any other reason, such as trauma or psoriasis.

\section{Disclosure Statement}

The authors report no relevant conflicts of interest.

\section{References}

1 Gupta AK, Ryder J, Summerbell RC: Comparison of efficacy criteria across onychomycosis trials: need for standardization. Int J Dermatol 2003;42:312-315.

2 Haugh M, Helou S, Boissel JP, Cribier BJ: Terbinafine in fungal infections of the nails: a meta-analysis of randomized clinical trials. $\mathrm{Br} \mathrm{J}$ Dermatol 2002;147:118-121.

3 Scher RK, Tavakkol A, Sigurgeirsson B, Hay RJ, Joseph WS, Tosti A, Fleckman P, Ghannoum $\mathrm{M}$, Armstrong DG, Markinson BC, Elewski BE: Onychomycosis: diagnosis and definition of cure. J Am Acad Dermatol 2007; 56:939-944.

4 Ghannoum M, Isham N, Catalano V: A second look at efficacy criteria for onychomycosis: clinical and mycological cure. Br J Dermatol 2014;170:182-187.

5 Shemer A, Sakka N, Baran R, Scher R, Amichai B, Norman L, Farhi R, Magun R, Brazilai A, Daniel R: Clinical comparison and complete cure rates of Terbinafine efficacy in affected onychomycotic toenails. J Eur Acad Dermatol Venereol 2015;29:521-526.

6 Gupta AK, Paquet M, Simpson F, Tavakkol A: Terbinafine in the treatment of dermatophyte toenail onychomycosis: a meta-analysis of efficacy for continuous and intermittent regimens. J Eur Acad Dermatol Venereol 2013;27: 267-272.

7 Shemer A: Update: medical treatment of onychomycosis. Dermatol Ther 2012;25:582-593.

8 Gupta AK, Ryder JE, Johnson AM: Cumulative meta-analysis of systemic antifungal agents for the treatment of onychomycosis. $\mathrm{Br}$ J Dermatol 2004;150:537-544.
9 Sigurgeirsson B, Billstein S, Rantanen T, Ruzicka T, di Fonzo E, Vermeer BJ, Goodfield MJ, Evans EG: L.I.ON. Study: efficacy and tolerability of continuous terbinafine (Lamisil) compared to intermittent itraconazole in the treatment of toenail onychomycosis. Lamisil vs. Itraconazole in Onychomycosis. Br J Dermatol 1999;141(suppl 56):5-14.

10 Sigurgeirsson B: Prognostic factors for cure following treatment of onychomycosis. J Eur Acad Dermatol Venereol 2010;24:679-684.

11 Fiallo P, Cardo PP: Age as limiting factor of the efficacy of itraconazole for treatment of onychomycosis. Mycoses 2001;44:191-194.

12 Sigurgeirsson B, Steingrimsson O: Risk factors associated with onychomycosis. J Eur Acad Dermatol Venereol 2004;18:48-51.

13 Feldstein S, Totri C, Friedlander SF: Antifungal therapy for onychomycosis in children. Clin Dermatol 2015;33:333-339.

14 Avner S, Nir N, Henri T: Fifth toenail clinical response to systemic antifungal therapy is not a marker of successful therapy for other toenails with onychomycosis. J Eur Acad Dermatol Venereol 2006;20:1194-1196.

15 Rigopoulos D, Papanagiotou V, Daniel R 3rd, Piraccini BM: Onychomycosis in patients with nail psoriasis: a point to point discussion. Mycoses 2017;60:6-10.

16 Warshaw EM, Nelson D, Carver SM, Zielke GR, Webster N, Lederle FA, Fett DD: A pilot evaluation of pulse itraconazole vs. terbinafine for treatment of Candida toenail onychomycosis. Int J Dermatol 2005;44:785-788.
17 Arca E, Tastan HB, Akar A, Kurumlu Z, Gur AR: An open, randomized, comparative study of oral fluconazole, itraconazole and terbinafine therapy in onychomycosis. J Dermato$\log$ Treat 2002;13:3-9.

18 Piepponen T, Blomqvist K, Brandt $\mathrm{H}$, Havu V, Hollmen A, Kohtamaki K, Lehtonen L, Turjanmaa K: Efficacy and safety of itraconazole in the long-term treatment of onychomycosis. J Antimicrob Chemother 1992;29: 195-205.

19 Carney C, Tosti A, Daniel R, Scher R, Rich P, DeCoster J, Elewski B: A new classification system for grading the severity of onychomycosis: Onychomycosis Severity Index. Arch Dermatol 2011;147:1277-1282.

20 Pajaziti L, Vasili E: Treatment of onychomycosis - a clinical study. Med Arch 2015;69: 173-176.

21 Bennett D, Rubin AI: Dermatophytoma: a clinicopathologic entity important for dermatologists and dermatopathologists to identify. Int J Dermatol 2013;52:1285-1287.

22 Burkhart CN, Burkhart CG, Gupta AK: Dermatophytoma: recalcitrance to treatment because of existence of fungal biofilm. J Am Acad Dermatol 2002;47:629-631.

23 Zaias N, Rebell G, Escovar S: Asymmetric gait nail unit syndrome: the most common worldwide toenail abnormality and onychomycosis. Skinmed 2014;12:217-223.

24 Zaias N, Rebell G, Casal G, Appel J: The asymmetric gait toenail unit sign. Skinmed 2012; 10:213-217. 